\title{
Erratum to: Fermentative production and direct extraction of (-)-a-bisabolol in metabolically engineered Escherichia coli
}

Gui Hwan Han ${ }^{1}$, Seong Keun Kim¹,2, Paul Kyung-Seok Yoon 1,3, Younghwan Kang ${ }^{1}$, Byoung Su Kim4, Yaoyao Fu', Bong Hyun Sung ${ }^{5}$, Heung Chae Jung ${ }^{1}$, Dae-Hee Lee ${ }^{1,2^{*}}$, Seon-Won Kim ${ }^{* *}$ and Seung-Goo Lee ${ }^{1,2^{*}}$

\section{Erratum to: Microb Cell Fact (2016) 15:185 DOI 10.1186/s12934-016-0588-2}

Upon publication of this article [1], it was brought to our attention that Figure 7 contained an error. $(-)-\alpha$-bisabolol is shown in $\mathrm{g} / \mathrm{L}$ and not $\mathrm{mg} / \mathrm{L}$ as incorrectly presented in the original version of the article. The correct figure is given in this erratum (Fig. 7).

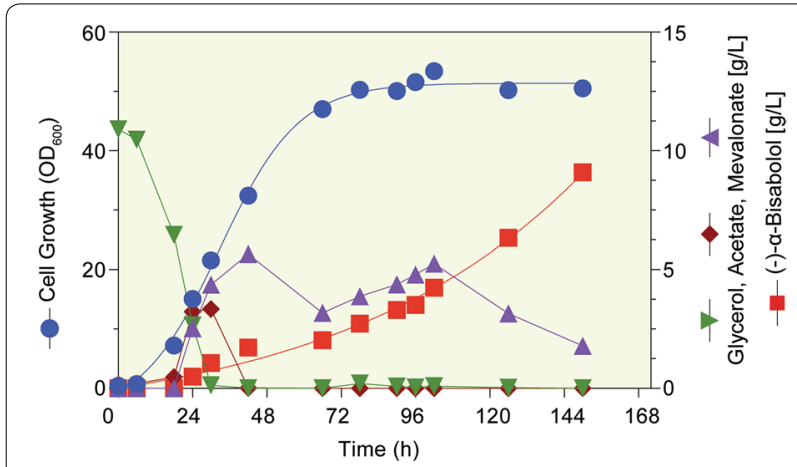

Fig. 7 Fed-batch fermentation of E. coli DH5a in TB medium supplemented with $10 \mathrm{~g} / \mathrm{L}$ of glycerol as initial carbon source. After complete depletion of glycerol, glycerol was fed at $3 \mathrm{~g} / \mathrm{L} / \mathrm{h}$. Concentrations of acetate, mevalonate, and glycerol were determined by HPLC and (-)-a-bisabolol content was measured using GC. Canola oil $10 \%(\mathrm{v} / \mathrm{v})$ instead of $n$-dodecane $20 \%(\mathrm{v} / \mathrm{v})$ was used to overlay $30 \mathrm{~L}$ $\mathrm{TB}$ medium in a $50 \mathrm{~L}$ fermenter

\begin{abstract}
Author details
1 Synthetic Biology and Bioengineering Research Center, Korea Research Institute of Bioscience and Biotechnology (KRIBB), Daejeon 34141, Republic of Korea. ${ }^{2}$ Biosystems and Bioengineering Program, University of Science and Technology (UST), Daejeon, 34113, Republic of Korea. ${ }^{3}$ Department of Chemical and Biological Engineering, Korea University, Seoul 02841, Republic of Korea. ${ }^{4}$ Department of Biotechnology, Chonnam National University, Yeosu 550749, Republic of Korea. ${ }^{5}$ Bioenergy and Biochemical Research Center, Korea Research Institute of Bioscience and Biotechnology (KRIBB), Daejeon 34141, Republic of Korea. ${ }^{6}$ Division of Applied Life Science (BK21 Plus), PMBBRC, Gyeongsang National University, Jinju 52828, Republic of Korea.
\end{abstract}

The online version of the original article can be found under doi:10.1186/s12934-016-0588-2.

Received: 19 January 2017 Accepted: 19 January 2017

Published online: 31 January 2017

\section{Reference}

1. Han GH, Kim SK, Yoon PKS, Kang Y, Kim BS, Fu Y, Sung BH, Jung HC, Lee D-H, Kim S-W, Lee S-G. Fermentative production and direct extraction of (-)-a-bisabolol in metabolically engineered Escherichia coli. Microb Cell Fact. 2016;15:185. doi:10.1186/s12934-016-0588-2.

\footnotetext{
*Correspondence: dhlee@kribb.re.kr; swkim@gnu.ac.kr; sglee@kribb.re.kr ${ }^{1}$ Synthetic Biology and Bioengineering Research Center, Korea Research Institute of Bioscience and Biotechnology (KRIBB), Daejeon 34141, Republic of Korea

${ }^{6}$ Division of Applied Life Science (BK21 Plus), PMBBRC, Gyeongsang National University, Jinju 52828, Republic of Korea

Full list of author information is available at the end of the article
} 